Relato de caso / Case report

\title{
Macroglobulinemia de Waldenström com hemorragia intra-ocular
}

\author{
Waldenström's macroglobulinaemia with intraocular hemorrhage
}

João J. Nassaralla Júnior ${ }^{1}$

Maria do Rosário F. Roberti ${ }^{2}$
O objetivo deste trabalho é relatar um caso de macroglobulinemia de Waldenström com envolvimento retiniano e da circulação coroidiana, com boa resposta ao tratamento. O desenho é um caso controle intervencional. Um homem de 47 anos foi encaminhado para o serviço de hematologia para avaliação de anemia e linfadenopatia inguinal. O diagnóstico de macroglobulinemia de Waldenström foi feito com base nos achados clínicos e laboratoriais. O paciente desenvolveu hemorragia intra-ocular sem associação com hipertensão e com fatores de coagulação normais. Exame fundoscópico revelou retardamento na circulação coriorretiniana, microaneurismas, e telangiectasias no pólo posterior. Angiografia fluorescente demonstrou o prolongamento do tempo da circulação coroidiana, tempo de trânsito arteriovenoso também prolongado, e áreas de não perfusão capilar. Estes resultados foram acompanhados por uma elevação acentuada da IgM. A maioria dos achados oculares foi revertida com a plasmaférese. A macroglobulinemia de Waldenström pode envolver a retina e a circulação coroidiana, porém pode ser reversível com o tratamento apropriado. Rev. bras. hematol. hemoter. 2006; 28(4):310-314.

Palavras-chave: Síndrome de hiperviscosidade; imunoglobulinas; mieloma múltiplo; macroglobulinemia de Waldenström.

\section{Introdução}

A macroglobulinemia de Waldenström (MW) foi descrita pela primeira vez por Waldenström, em 1944.,1,2 A MW é uma entidade clínico-patológica distinta, de etiologia desconhecida, resultante da proliferação clonal de linfócitos B maduros com diferenciação plasmocítica. Estes resultam em um infiltrado da medula óssea que produzem componente monoclonal, a imunoglobulina M (IgM). ${ }^{3,4}$ Na classificação REAL corresponde ao linfoma linfoplasmocítico. ${ }^{5}$ Os critérios diagnósticos incluem a presença de gamopatia monoclonal IgM, infiltração da medula óssea por linfócitos, células plasmocitóides e plasmócitos, associados a padrão difuso, intersticial ou nodular e imunofenótipo Ig+CD5-CD10CD19+CD20+CD23-. Em 5,7\% dos casos observamos a ocor- rência da síndrome de hiperviscosidade. ${ }^{6}$ Esta rara doença possui importante espectro de sinais e complicações. ${ }^{1,3}$ Neste estudo relatamos o caso de um homem portador de WM com hemorragia intra-ocular. Poucos casos são relatados na literatura internacional. ${ }^{2,3,7-9}$

\section{Descrição do caso}

Em dezembro de 1996, um homem de 42 anos procurou serviço de hematologia para investigação de anemia. Apresentava fraqueza nesta época. O hemograma inicial mostrava anemia e plaquetopenia (Tabela 1). Foi submetido à biópsia de medula, sendo encontrada infiltração linfoplasmocitóide com padrão nodular e fibrose reticulínica leve. A biópsia do linfonodo inguinal apresentou neoplasia maligna com exu-

${ }^{1}$ Doutor em Ciências da Saúde pela UnB. Doutor em Oftalmologia pela UFMG. Professor da pós-graduação da UnB.

Departamento de Retina, Vítreo e Uveítes do Instituto de Olhos de Goiânia.

${ }^{2}$ Doutora em Hematologia pela USP. Departamento de Hematologia do Hospital Geral de Goiânia. Professora da disciplina de

Práticas Integradoras I da UFG.

Trabalho realizado no Departamento de Retina e Vítreo do IOG.

Correspondência: João Nassaralla

R. L, 53 \# 1200 - Setor Oeste

74120-050 - Goiânia-GO - Brasil

E-mail:nassaral@terra.com.br 
Tabela 1

Hemograma do paciente ao diagnóstico e no momento da última recidiva.

\begin{tabular}{ccc}
\hline & $12 / 1996$ & $02 / 2006$ \\
\hline Leucócitos & $4,2 \times 10^{9} / \mathrm{L}$ & $2,6 \times 10^{9} / \mathrm{L}$ \\
Neutrófilos & $2,7 \times 10^{9} / \mathrm{L}$ & $1,2 \times 10^{9} / \mathrm{L}$ \\
Linfócitos & $1,2 \times 10^{9} / \mathrm{L}$ & $9,1 \times 10^{9} / \mathrm{L}$ \\
Plaquetas & $120 \times 10^{9} / \mathrm{L}$ & $56 \times 10^{9} / \mathrm{L}$ \\
Hemoglobina & $96 \mathrm{~g} / \mathrm{L}$ & $80 \mathrm{~g} / \mathrm{L}$ \\
\hline
\end{tabular}

Tabela 2

Protocolos de quimioterapia empregados no tratamento

\begin{tabular}{|c|c|c|c|c|c|c|}
\hline Sigla & $\begin{array}{c}\text { Drogas } \\
\text { utilizadas }\end{array}$ & $\begin{array}{c}\text { Doses } \\
\left(\mathrm{mg} / \mathrm{m}^{2}\right)\end{array}$ & Via & & $\begin{array}{c}\mathrm{N}^{\circ} \\
\text { ciclos }\end{array}$ & $\begin{array}{c}\text { Data do } \\
\text { tratamento }\end{array}$ \\
\hline \multirow{4}{*}{$\begin{array}{c}\mathrm{M}_{2} \\
\text { modificado } 25\end{array}$} & VCR & 1,2 & EV & D1 & \multirow{4}{*}{6} & \multirow{4}{*}{$12 / 1996$} \\
\hline & MEL & 8 & VO & D1-4 & & \\
\hline & CFM & 400 & EV & D1 & & \\
\hline & PDN & 40 & VO & D1-7 & & \\
\hline Cladribina $^{26}$ & $2 \mathrm{CdA}$ & $0,1^{*}$ & EV & D1-5 & 6 & 09/1998 \\
\hline $\mathrm{CBL}^{27}$ & CBL & $0,1^{\star \star}$ & VO & contínuo & 10 & $11 / 1999$ \\
\hline \multirow{5}{*}{$\mathrm{R}-\mathrm{CHOP}^{28}$} & CFM & 650 & EV & D1 & \multirow{5}{*}{4} & \multirow{5}{*}{$11 / 1999$} \\
\hline & ADRIA & 50 & EV & D1 & & \\
\hline & VCR & $2^{\star \star \star}$ & EV & D1 & & \\
\hline & PDN & 60 & VO & D1-5 & & \\
\hline & Rituximab & & EV & Do & & \\
\hline \multirow{3}{*}{$\mathrm{COP}^{29}$} & CFM & 750 & EV & D1 & \multirow{3}{*}{3} & \multirow{3}{*}{$12 / 2005$} \\
\hline & $\mathrm{VCR}^{\star \star}$ & 2 & EV & D1 & & \\
\hline & PDN & 40 & VO & D1-5 & & \\
\hline $\mathrm{CBL}^{27}$ & CBL & $0,1^{\star \star}$ & VO & contínuo & 3 & 03/2006 \\
\hline
\end{tabular}

CFM: ciclofosfamida, ADRIA: doxorrubicina, VCR: vincristina, MEL: melfalan, PDN: prednisona, CBL: clorambucil, 2CdA: cladribina, R: Rituximabe.

*0,1mg/k contínuo; *^2 mg dose máxima

berante diferenciação plasmocítica e a imuno-histoquímica deste material revelou tratar-se de neoplasia linfóide B com monoclonalidade para cadeia? Por dificuldades técnicas, o imunofenótipo não pode ser determinado por citometria de fluxo. A IgM sérica era de 9,91 g/dL. Embora com níveis elevados, não apresentava sintomas de hiperviscosidade. Foi submetido a seis ciclos do protocolo M2 modificado (Tabela 2) com melhora das citopenias e dos níveis de IgM sérica. De setembro de 1997 até novembro de 1999 foi tratado com outros esquemas de quimioterapia apresentando melhora parcial dos níveis de IgM, sendo o menor valor encontrado 2,54 g/dL. Em julho de 2003 apresentou borramento visual sendo constatada hemorragia retiniana. Nesta época, a IgM sérica era de 10,40g/dL. Foi submetido a dois ciclos de plasmaférese terapêutica com troca de uma volemia e reposição com soro albuminado, seguido de quatro ciclos de R-CHOP (Tabela 2). Houve melhora dos sintomas oculares e dos níveis de IgM (7,8 g/dL) tendo o pacien- te abandonado o tratamento até janeiro de 2006, quando procurou o serviço de hematologia com queixa de gengivorragia, epistaxe e borramento visual em olho D a um mês. Apresentava exames de coagulação normais e pancitopenia (Tabela 1). A dosagem da IgM sérica era de 10,7 g/dL. O exame do olho direito apresentou baixa acuidade visual (20\50cc). A visão do olho esquerdo estava dentro dos padrões da normalidade (20\20cc). O exame ocular mostrou alterações na papila do nervo óptico com aumento discreto da escavação (0.4 em $\mathrm{AO})$, tortuosidade vascular venosa e hemorragias retinianas (Figura 1). No olho direito as hemorragias acometiam a região macular (Figura 2), com grande área (10DP) de atrofia na periferia inferior da coriorretina (Figura 3). A pressão ocular estava dentro dos padrões da normalidade $(12 \mathrm{mmHg}$ em ambos os olhos). Ao exame biomicroscópio apresentou in-

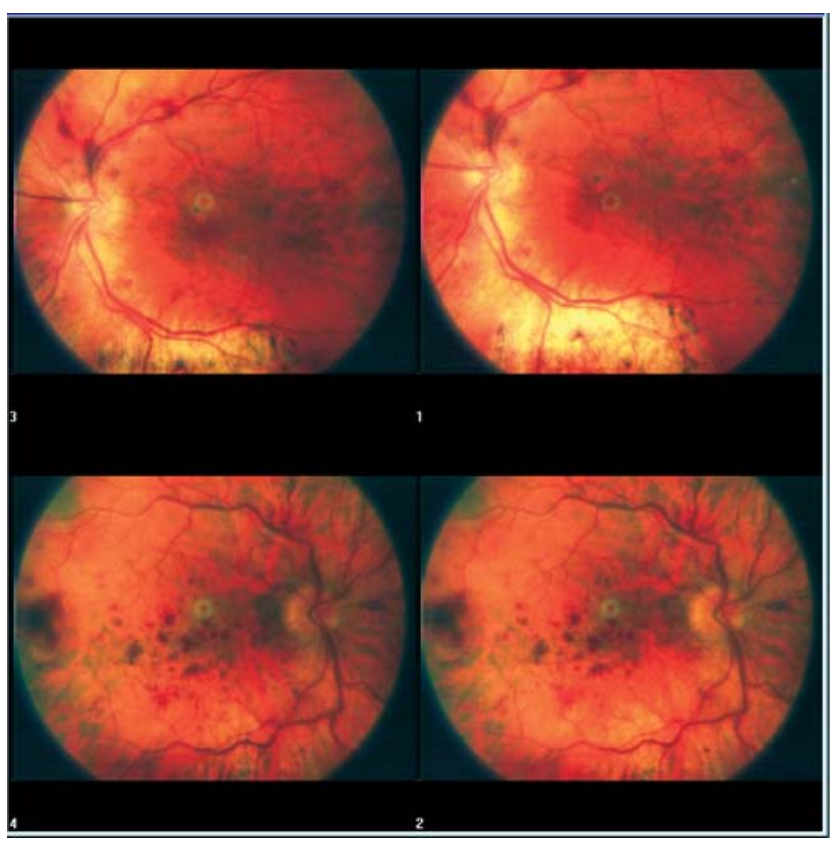

Figura 1. Retinografia apresentando hemorragias retinianas, sendo que no olho direito ( 4 e 2) as hemorragias são maculares. No olho esquerdo nota-se área de atrofia retiniana inferiormente

Figura 2. Retinografia fluorescente do olho direito apresentando vasos ensalsichados e hipofluorescência nos microaneurismas e nas áreas de hemorragias na mácula

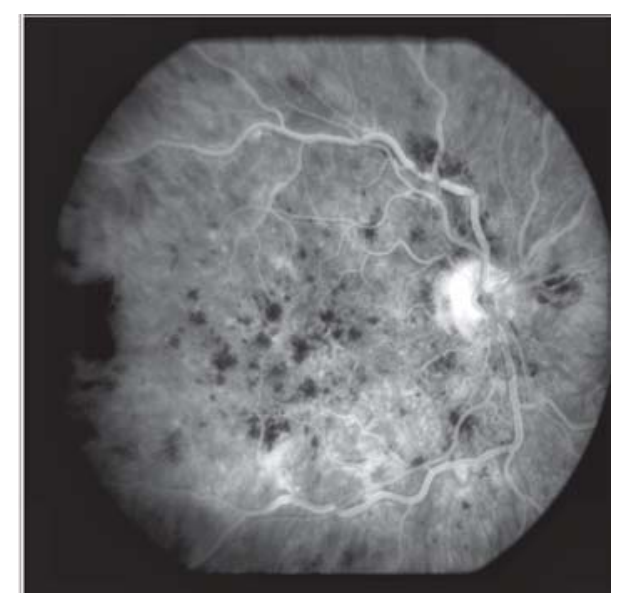


jeção conjuntival discreta. No exame de angiografia fluoresceínica apresentou aumento do tempo braço-retina (18 segundos), fluxo vascular do contraste retardado com 20 minutos de fluorescência após a injeção do mesmo. Áreas de hipofluorescência nas regiões de hemorragias retinianas, além de microaneurismas no pólo posterior em ambos os olhos (Figuras 2 e 3). Foram realizadas duas sessões de plasma-

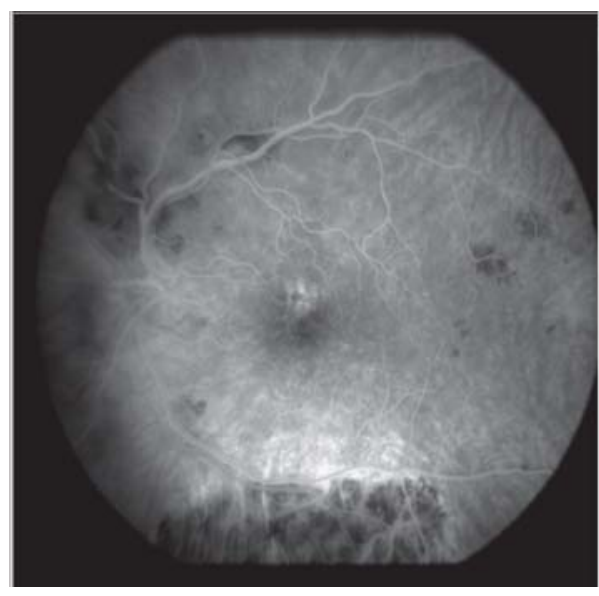

Figura 3.

Retinografia

fluorescente do olho esquerdo apresentando hipofluorescência na área de atrofia retiniana, inferiormente e microaneurismas na região suprafoveal

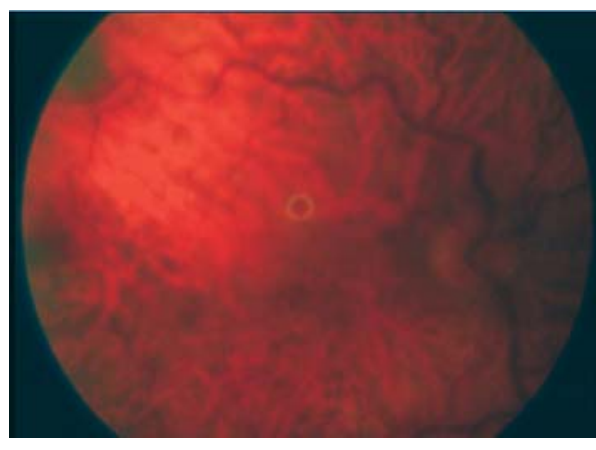

Figura 4.

Retinografia do olho direito após o tratamento apresentando vasos tortuosos, mostrando a remissão das hemorragias e microaneurismas na região do pólo posterior férese terapêutica com troca de uma volemia e reposição com soro albuminado a $5 \%$ e quimioterapia paliativa com COP (Tabela 2).

Após três meses do início do tratamento, o paciente retornou para reavaliação oftalmológica com melhora da visão do olho direito (cc 20/30) e manutenção da visão do olho adelfo (cc 20/20). O exame de fundo de olho manteve a tortuosidade vascular e as cicatrizes de coriorretinite inalteradas, porém sem as hemorragias retinianas que foram absorvidas. Ao biomicroscópio não apresentou alterações assim como a pressão ocular. No exame de retinografia e retinografia fluoresceínica (Figura 4 e 5) apresentou tempo braço-retina (13 segundos), fluxo vascular do contraste com 12 minutos de fluorescência após a injeção do mesmo. Fluorescência habitual nas regiões que anteriormente apresentavam hemorragia retiniana, com manutenção de microaneurismas e tortuosidade vascular evidente.

\section{Discussão}

A MW é uma doença infreqüente que contribui com cerca de $2 \%$ de todas as malignidades hematológicas. ${ }^{4,10} \mathrm{~A}$ média de idade é de 63 anos, com leve predominância do sexo masculino. ${ }^{4,10}$ Fraqueza, anorexia e perda de peso são os sinais comumente encontrados. ${ }^{4,10} \mathrm{O}$ fenômeno de Raynaud e sintomas resultantes de neuropatia periférica podem preceder o diagnóstico. Hepatomegalia, esplenomegalia ou adenomegalia são achados infreqüentes. ${ }^{4}$ Dentre os achados laboratoriais, a anemia é usualmente encontrada, podendo ser resultante da infiltração medular, diminuição da sobrevida das hemácias, paralisação da eritropoese, expansão do volume plasmático e sangramentos, principalmente do tubo digestivo. ${ }^{4}$ A leucometria e o número de plaquetas geralmente são normais ao diagnóstico. ${ }^{4,10}$ A eletroforese de proteínas séricas revela pico monoclonal devido à produção de IgM e a cadeia leve nesses casos, em 75\% é $\kappa .{ }^{4}$

Perdas cromossômicas numéricas en-

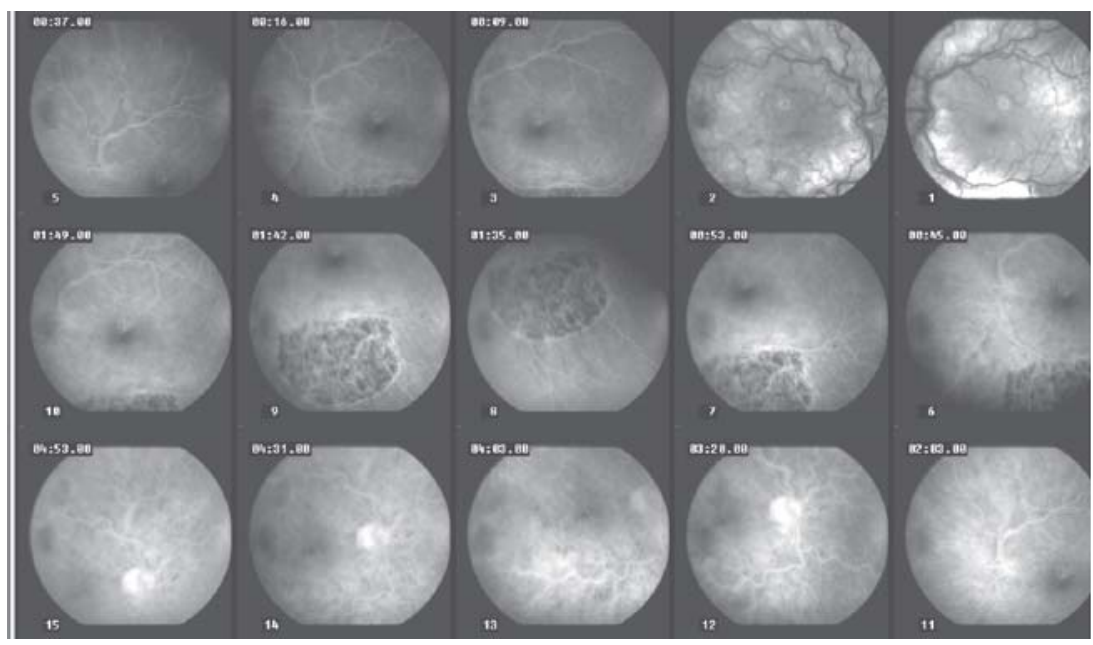

Figura 5. Retinografia fluorescente de ambos os olhos apresentando hipofluorescência na área de atrofia retiniana, sem a presença de hemorragias retinianas, evidenciando apenas microaneurismas suprafoveais no olho esquerdo volvendo os cromossomos 17,18, 19, 20,21,22, X e Y e ganhos nos cromossomos 3, 4 e 12 são descritos. A deleção 6q é observado em $40 \%$ a $90 \%$ dos casos. ${ }^{4}$ Embora a anormalidade 14q32 seja comum em linfomas de célula $B$, nenhum caso desta anomalia foi encontrado no estudo de Avet-Loiseau e cols. A ausência de rearranjamento IgH observada na MW pode auxiliar no diagnóstico diferencial entre MM secretor de IgM.,11

A produção da IgM não é característica somente da MW. Outros linfomas de baixo grau, tais como linfoma da zona marginal ou linfoma linfocítico de pequenas células podem estar associados à produção de IgM. ${ }^{5}$ As características imunofenotípicas destes linfócitos incluem a expressão de marcadores 
pan-B (CD19, CD20, CD22). ${ }^{6,12}$ A expressão do CD5, CD10 e CD23 são achados incomuns, ${ }^{4,6}$ embora alguns autores relatarem positividade para o CD23. ${ }^{12,13} \mathrm{Em}$ casos onde a infiltração da medula óssea é exclusiva de plasmócitos e o imunofenótipo demonstre IgM+CD20-CD138+ não há critérios para MW e deverão ser estes casos, considerados mieloma múltiplo (MM). ${ }^{6}$

A síndrome de hiperviscosidade causada por aumento paraproteínas tem como principal etiologia a MW, seguida de MM secretor de IgA e IgG14. Entre 30\% a 70\% dos portadores de MW desenvolvem a síndrome de hiperviscosidade que se manifesta por epistaxe, borramento visual, dispnéia e cefaléia. ${ }^{15}$ Os sintomas geralmente aparecem quando os níveis de IgM sérica estão acima de $3 \mathrm{~g} / \mathrm{dL}$, que corresponde a nível de viscosidade de $5 \mathrm{cp}$ (valores normais: 1.4 a $1.8 \mathrm{cp}) .{ }^{13}$ A medida da viscosidade sérica não é uma prática comum, porém o exame do fundo do olho simples ou retinografia fluorescente é um método sensível para diagnosticar a síndrome de hiperviscosidade onde hemorragias retinianas, papiledema, distensão e dilatação de vasos (forma de salsicha) podem ser detectados. ${ }^{1-3,8,10,13,16}$

Anemia é o achado mais comum em pacientes sintomáticos. O curso clínico é bastante heterogêneo, porém geralmente é uma doença de curso insidioso, com sobrevida média de sete anos. A causa mais comum de óbito é infecção ou segunda malignidade. ${ }^{7}$ Complicações neurológicas, principalmente neuropatia periférica, associados ao componente monoclonal pode ser evidenciado. ${ }^{1,7}$ Hemorragias, principalmente do trato gastrointestinal, têm sido descritas na literatura. ${ }^{1,7,17}$

Entre os achados oculares podemos destacar a celulite orbitária, secundária a sinusite e injeção conjuntival, podendo-se inclusive observar, na biomicroscopia, segmentação dos eritrócitos. ${ }^{1,5,16,18-20}$ Também podem ser observados cristais na córnea e conjuntiva, sendo que estes cristais apresentam reação com as imunoglobulinas, quando submetidos a imunofluorescência e imunoperoxidase. ${ }^{2,7,16,18-20}$ Alterações retinianas são encontradas em 50\% dos pacientes com MW. 2,7,20,21 Classicamente, alterações venosas como dilatação e tortuosidade vascular são encontradas, provavelmente devido à hiperviscosidade sangüínea e a dificuldade no fluxo. Este aumento da congestão pode levar à hemorragia retiniana em chama de vela, além do edema retiniano, edema papilar, microaneurismas e oclusão vascular. ${ }^{2,5,14,16,18-20}$ Alguns autores descrevem os achados oculares de forma simétrica e bilateral. ${ }^{18} \mathrm{O}$ caso aqui relatado apresentava microaneurismas, hemorragia retiniana e vítrea somente no olho direito, tortuosidade venosa (mais acentuada no olho direito) sem os usuais fatores de risco, tal como hipertensão arterial, que pode explicar a base da síndrome de hiperviscosidade, quando uma excessiva produção de paraproteína leva ao aumento da viscosidade plasmática causando a redução do fluxo sangüíneo, estase vascular, oclusão e isquemia com eventual alteração da função plaquetária. ${ }^{21,22}$
Os microaneurismas estavam presentes em ambos olhos como descrito por alguns autores, ${ }^{18}$ porém no nosso caso não houve remissão completa dos mesmos.

O tratamento não deve ser baseado nos níveis de IgM sérica, uma vez que esta pode não corresponder às manifestações clínicas da MW. Considera-se razoável tratar aqueles pacientes com aumento laboratorial documentado da IgM e sinais e sintomas progressivos da doença. Nos pacientes com anemia ( $\geq 10 \mathrm{~g} / \mathrm{dL}$ ) e/ou plaquetopenia (< que $100 \mathrm{x}$ $10^{9} / \mathrm{L}$ ), secundários à infiltração medular, adenomegalia significativa (bulky), organomegalia ou sintomas constitucionais, também devem ser instituída terapêutica. Complicações como a síndrome de hiperviscosidade, neuropatia periférica, amiloidose sistêmica, insuficiência renal ou crioglobulinemia sintomática podem também ser indicativas de tratamento. Na ausência dos fatores acima descritos é razoável apenas o seguimento clínico. Reavaliações trimestrais destes pacientes, com dosagem da IgM sérica é recomendado. Nos pacientes estáveis o seguimento pode ser anual., ${ }^{4,23}$

As opções iniciais de tratamento são agentes alquilantes, em geral clorambucil, análogos da purina (fludarabina ou cladribina) e terapia com anticorpo monoclonal como o rituximabe. ${ }^{4,13,23}$ Nos pacientes com síndrome de hiperviscosidade o tratamento de suporte com plasmaférese terapêutica, com troca de uma volemia e retorno com soro albuminado a 5\% é o recomendado., ${ }^{4,17,23,25}$ Usualmente, uma seção de plasmaférese, com troca de uma volemia reduz drasticamente o nível sérico de IgM, que é de circulação intravascular, resultando em pronta melhora da sintomatologia. ${ }^{14,24}$ O método de centrifugação automatizada, com retorno de soro albuminado a $5 \%$ parece mais eficaz que aqueles que utilizam membranas de separação, filtração ou imunoadsorção. O retorno com plasma fresco congelado pode ser necessário nos pacientes com alterações nos tempos da coagulação. ${ }^{15}$ Nos casos com complicações oculares, o uso da plasmaférese tem resultado em reversão da sintomatologia. ${ }^{14,17}$ Em nosso caso houve melhora dos sintomas hemorrágicos, remissão dos microaneurismas, assim como a queixa ocular. Persistindo somente a tortuosidade vascular, mesmo que de forma menos acentuada, como é relatado na literatura. ${ }^{18,20}$

A MW é uma doença rara, e o exame do fundo de olho é capaz de detectar alterações oculares, mesmo nos casos assintomáticos, achamos prudente avaliação semestral pelo oftalmologista e pronta instituição terapêutica nos casos onde haja alterações no fundo de olho. Assim conseguimos a involução da maioria dos sinais oculares e melhora e normalização da acuidade visual. Sendo que o tratamento precoce é fator preditivo para se evitar outras alterações sistêmicas.

\section{Abstract}

The aim of this work is to report a case of Waldenström's Macroglobulinaemia involving both retinal and choroidal circulation that resolved after treatment. The design is an interventional case 
report. A 47-year old male was admitted for evaluation of anaemia, and groin lymphadenopathy. A diagnosis of Waldenström's macroglobulinaemia was made based on clinical and laboratory findings. The patient developed intraocular hemorrhage without associated hypertension and with normal coagulation profile. A funduscopic examination revealed marked dilation and beading of the venous system, microaneurysms, and telangiectatic capillary beds in the posterior pole. A fluorescein angiography showed delayed choroidal filling, prolonged arteriovenous transit time and areas of capillary non-perfusion. These findings were accompanied by a severe IgM increase in serum viscosity. The most important ocular findings were reversible after plasmapheresis. Waldenström's macroglobulinaemia can involve both retinal and choroidal circulation. The prominent microvasculopathy is reversible after appropriate treatment. Rev. bras. hematol. hemoter. 2006; 28(4): 310-314.

Key words: Waldenström's macroglobulinaemia; immunoglobulins; multiple myeloma.

\section{Referências Bibliográficas}

1. MacKenzie MR, Fudenberg HH. Macroglobulinemia: An analysis for forty patients. Blood 1972; 39:874-89.

2. Gold DH, Weingeist TA. The eye in systemic disease. Philadelphia: J.B. Lippincott; 1986. pp135-6.

3. Spalter HF. Abnormal serum protein and retinal vein trombosis. Arch Ophthalmol 1959;62:868-81.

4. Gertz MA, Merlini G, Treon SP. Amyloidosis and Waldenström's Macroglobulinemia. Hematology 2004;257-82.

5. Harris NL, Jaffe ES, Diebold J, Flandrin G, Muller-Hermelink HK, Vardiman J, et al. The World Health Organization classification of neoplastic diseases of haematopoietic and lymphoid tissues: report of the clinical advisory committee meeting, Airlie House, Virginia, November 1997. Histopathology 2000;36:69-87

6. Owen RG. Developing diagnostic criteria in Waldenström's macroglobulinemia. Sem Oncol 2003;30:196-200.

7. Thomas EL, Olk RJ, Markman M, et al. Irreversible visual loss in Waldenström's macroglobulinaemia. Br J Ophthalmol 1983;67:102-6.

8. Karmarkar T, Saha A, Verma N, Walker R, Sakhuja V, Datta U. Waldenström's macroglobulinemia: a case with lymphocytosis. J Assoc Physicians India 1993;41:458-9

9. Lekhra OP, Sawhney IM, Gupta A, Varma S, Chopra JS. Venous retinopathy in Waldenström's macroglobulinemia. J Assoc Phys Ind 1996;44:61-2.

10. Tricot G. Multiple Myeloma and other plasma cell disorders. In: Hoffman R, Benz EJ Junior, Shattil SJ, Furie B, Cohen HJ, Silberstein LE, McGlave P, editors. Hematology Basic Principles and Practice. 4th Ed. Elsevier, 2005. pp 1521-3.

11. Avet-Loiseau H, Garand R, Lodé L, RobillardN, Bataille R. 14q32 translocations discriminate IgM Multiple Myeloma from Waldenström's macroglobulinemia. Sem Oncol 2003;30:153-5.

12. Konoplev S, Medeiros LJ, Bueso-Ramos CE, Jorgensen JL, Lin P. Immunophenotypic profile of lymphoplasmocytic lymphoma/ Waldenström's Macroglobulinemia. Am J Clin Pathol 2005;124: 414-20.

13. Dimopolos MA, Panayiotidis P, Moulopoulos LA, Sfikakis P, Dalakas M. Waldenström's macroglobulinemia: Clinical features, complications and management. J Clin Oncol 2000;18:214-26.

14. Zarkovic M, Kwaan HC. Correction of hyperviscosity by apheresis. Sem Thromb Hemost 2003;29:535-42.
15. Drew MJ. Plasmapheresis in dysproteinemias. Ther Apher 2002; 6:45-52.

16. Rezai KA, Patel SC, Eliott D, Becker MA. Rheumatoid hyperviscosity syndrome: reversibility of microvascular abnormalities after treatment. Am J Ophthalmol 2002;134:130-2.

17. Tumors of lymphoid tissues: the paraproteinemias. In: Penington D, Rush B, Castaldi P, editors. GC de Gruchy Clinical Haematology in Medical practice. 4th Ed. Delhi: CBS Publishers 1985. pp 502-53.

18. Facon T, Brouillard M, Duhamel A, et al. Prognostic factors in Waldenström's macroglobulinemia: A report of 167 cases. J Clin Oncol 1993;11:1.553-8,

19. Carr RE, Henkind P. Retinal findings associated with serum hyperviscosity. Am J Ophthalmol 1963;56:23-31.

20. Sarnat RL, Jampol LM. Hyperviscosity retinopathy secondary to polyclonal gammopathy in a patient with rheumatoid arthritis. Ophthalmology 1986;93:124-7.

21. Mc Callister BD, Bayrd ED, Harrison EG, Mc Guckin WF. Primary Macroglobulinemia: review with a report of thirty-one cases and notes on the value of continuous chlorambucil therapy. Am J Med 1967;43:394-434.

22. MacKenzie MR, Lee TK. Blood viscosity in Waldenström's macroglobulinaemia. Blood 1977;49:507-10.

23. Kyle RA, Treon SP, Alexanian R, Barlogie B, Björkholm M, et al. Prognostic markers and criteria to initiate therapy in Waldenström's Macroglobulinemia: Consensus panel recommendations from the second international workshop on Waldenström's macroglobulinemia. Sem Oncol 2003;30:116-20.

24. Siami GA, Siami FS. Plasmapheresis and paraproteinemia: cryoprotein-induced diseases, monoclonal gammopathy, Waldenström's macroglobulinemia, hyperviscosity syndrome, multiple myeloma, light chain disease, and amyloidosis. Ther Apher 1999;3:8-19.

25. Oken MM, Harrington DP, Abramson N, Kyle RA, Knospe W, et al. Comparison of melphalan and prednisone with vincristine, carmustine, melphalan, cyclophosphamide and prednisone in the treatment of multiple myeloma. Cancer 1999;86:957-68.

26. Betticher DC, Schmitz S-FH, Ratschiller D, von Rohr A, Egger T, et al. Cladribine (2-CDA) given as subcutaneous bolus injection is active in pretreated Waldenström's macroglobulinaemia. Br J Haematol 1997;99:358-63.

27. Kyle RA, Greipp PR, Gertz MA, Witzig TE, Lust JA, et al. Waldenström's macroglobulinaemia: a prospective study comparing daily with intermittent oral chlorambucil. Br J Haematol 2000; 108:737-42.

28. Zinzani PL, Pulsoni A, Perrotti A, Soverine S, Zaja F, et al. Fludarabine plus mitroxantrone with and without rituximab versus CHOP with and without rituximab as front-line treatment for patients with follicular lymphoma. J Clin Oncol 2004;22:1-8.

29. Klasa RJ, Meyer RM, Shustik C, Sawka CA, Smith A, et al. Randomized phase III study of Fludarabine Phosphate versus cyclophosphamide, vincristine, and prednisone in patients with recurrent low-grade non-Hodgkin's Lymphoma previously treated with alkylanting agent or alkylator-containing regimen. J Clin Oncol 2002;20:4.649-54.

Avaliação: Editor e dois revisores externos Conflito de interesse: não declarado

Recebido: 17/05/2006

Aceito: 25/10/2006 